\title{
Rursus
}

Russus

Poiétique, réception et réécriture des textes antiques

9 | 2016

Commentaires anciens (pragmatique \& rhétorique)

\section{Le lectorat de Donat, commentateur de Térence}

Who is the reader of Donatus' Comment on Terence?

\section{Bruno Bureau et Christian Nicolas}

\section{(2) OpenEdition}

Journals

Édition électronique

URL : http://journals.openedition.org/rursus/1222

DOI : 10.4000/rursus. 1222

ISSN : 1951-669X

Éditeur

Université Nice-Sophia Antipolis

\section{Référence électronique}

Bruno Bureau et Christian Nicolas, «Le lectorat de Donat, commentateur de Térence », Rursus [En ligne], 9 | 2016, mis en ligne le 28 juillet 2016, consulté le 01 mai 2019. URL : http:// journals.openedition.org/rursus/1222 ; DOI : 10.4000/rursus.1222

Ce document a été généré automatiquement le 1 mai 2019.

Rursus 


\title{
Le lectorat de Donat, commentateur de Térence
}

Who is the reader of Donatus' Comment on Terence?

\author{
Bruno Bureau et Christian Nicolas
}

1 Reconstruire la figure du lectorat des commentaires grammaticaux et le type même de lecture que ces textes impliquent suppose que l'on prenne en compte à la fois la nature très particulière de ces textes et la fonction qu'on peut leur assigner ${ }^{1}$. Par leur nature de littérature au second degré, et leur transmission souvent perturbée par la réduction de textes suivis en scholies marginales ou interlinéaires ${ }^{2}$, les commentaires proposent au lecteur une interaction différente de celle offerte par les textes littéraires, mais encore faut-il parvenir à dessiner même sous forme d'esquisse ou de silhouette cette figure du lecteur. Pour qui le commentateur écrit-il ? Quel niveau de prérequis est-il attendu du lecteur pour que les interactions supposées par le texte fonctionnent? Comment le lecteur parvient-il à construire sa lecture du commentaire et, à partir du commentaire, sa lecture même de l'œuvre commentée?

2 Pour répondre à ces questions, au moins en partie, pour ce qui concerne le commentaire spécifique attribué à Aelius Donat aux comédies de Térence, il faut d'abord reconstruire ce que pouvait être l'horizon d'attente d'un lecteur du commentaire, la figure du lecteur idéal que suppose l'auteur, avant de voir comment Donat met en scène ce lecteur dans son propre texte et ce qu'il suppose comme activité lectoriale quand il invite son public à le « lire ».

\section{Le lectorat du commentaire en dehors de Donat}

\section{1 choix du corpus et but de la comparaison}

3 Le plus simple aurait sans doute été de comparer Donat à Servius et aux autres représentants de la tradition du commentaire grammatical lemmatisé. Pourtant ce n'est pas ce que nous allons faire, en raison de la transmission même du texte de Donat, 
démembré en scholies marginales puis recomposé comme un tout. L'intérêt de la comparaison est à notre sens de rapporter le matériau de Donat à des commentaires qui n'ont précisément pas été démembrés, pour voir si dans ce que nous avons conservé de Donat, il demeure encore des éléments qui étaient sans doute plus clairs dans la version primitive. Nous avons donc choisi de faire porter notre étude sur le lectorat dans deux commentaires suivis complets et non démembrés, les Interpretationes Vergilianae de Tiberius Donat ${ }^{3}$, et le commentaire du de Cassiodore ${ }^{4}$. Ce qui nous intéresse évidemment, ce sont les éléments communs qui construisent une figure du " lecteur de commentaires » à comparer avec ce que nous lisons chez Donat.

Nous procéderons en deux temps: 1- quelle figure du lecteur construisent les commentaires; 2-comment et pourquoi le lecteur est-il convoqué et pour quelles interactions avec le commentateur.

\section{2 quelle figure de lecteur?}

\subsection{1 pourquoi lire le commentaire}

5 Tiberius Donat fournit une indication extrêmement claire sur le lectorat attendu et ses motivations dans l'ouverture du commentaire. La lecture du texte commenté est éclairée par le commentateur afin de fournir au lecteur à la fois un matériau technique grammatical et rhétorique et de dégager les enseignements moraux et sociaux du texte :

Claud. Don., prooem. p.6, GEORG.: hoc quoque mirandum debet aduerti, sic Aeneae laudem esse dispositam, ut in ipsam exquisita arte omnium materiarum genera conuenirent. Quo fit ut Vergiliani carminis lector rhetoricis praeceptis instrui possit et omnia uiuendi agendique officia reperire.

Voici encore une chose admirable qu'il faut remarquer : l'éloge d'Enée est composé de manière à ce qu'y convergent avec un art parfait toutes sortes de matériaux. C'est ce qui fait que le lecteur du poème virgilien peut s'instruire des règles de l'art oratoire tout en trouvant tout ce qu'il lui faut pour bien vivre et bien agir.

Pour confirmer cela, Cassiodore propose d'ailleurs à ses lecteurs une réinterprétation de l'idée que l'on trouvait chez Tibérius: ce n'est pas en lisant les classiques et leurs commentaires et en s'instruisant à leur artes que l'on trouvera cela, mais bien en lisant les psaumes et son commentaire à lui :

Cassiod. Ps. 39, 1. 127 : numquid tale est scenicas audire tragoedias, quale est in choris ecclesiae salutiferas cognoscere psalmodias? Istas res potius legentes spectare debemus, quas nec fatue quaerimus et proficue semper audimus. Dicendo enim quis similis tibi, malarum rerum arguit inuentores, qui se nequiter efferentes, fallacium artium auctores esse gloriantur

Eh bien, est-ce la même chose d'écouter des tragédies au théâtre et de connaître en faisant partie du chœur le salut que donne la psalmodie? Voici ce que dans nos lectures nous devons le plus avoir en vue : ce dont la recherche n'est pas vaine et dont l'écoute est toujours profitable. Car, en disant 'qui est semblable à toi', il confond les inventeurs de savoirs mauvais qui se montent la tête dans leur malignité en se faisant un titre de gloire du fait qu'on leur doive des arts trompeurs.

Le lecteur n'est donc pas seulement un «scolaire », qui lit par devoir, il est quelqu'un qui lit pour nourrir sa propre expérience de la méditation des textes. Cela induit une réflexion sur les prérequis attendus du lecteur. 


\subsection{2 les prérequis du lectorat}

8 Ni pour Tibérius Donat ni pour Cassiodore, le lecteur n'est prioritairement un débutant. Il a besoin de savoirs particuliers et d'une attitude particulière face au texte.

9 Le fils de Tibérius, Tiberianus, a déjà lu des commentaires ou se propose d'en lire et l'ouvrage de Tibérius vise à se situer en complément de ces lectures; le commentaire suppose donc pour être compris que l'on a devant soi autre chose que des inscii lectores (Claud. Don. 10, p. 321 GEORG.). La nature des connaissances que doit posséder le lecteur repose à la fois sur un fonds culturel et sur une forme de fréquentation naïve du texte à commenter. Ainsi, Tibérius considère que le lecteur doit savoir un peu de mythologie et qu'il doit avoir également observé quelques habitudes du poète, parce que le commentateur se fonde sur ces compétences simples de son lecteur pour lui proposer autre chose. Par exemple, le lecteur ne doit pas s'attendre à ce qu'on lui parle de la nuit, parce qu'il doit savoir que Virgile abrège ce type de notation; et le commentateur attire au contraire son attention sur le commentaire du lever du jour :

Claud. Don. 11, p. 433 GEORG.: multis locis poeta lucescentis dies describit, de noctibus tacet consulens breuitati dictionis, quae legentibus familiaris est.

En de nombreux passages le poète donne une description du jour qui se lève sans rien dire des nuits, car il veille à une certaine brièveté dans son style qui est familière aux lecteurs.

10 De même si Cassiodore prétend qu'il s'adresse à des gens qui ne connaissent pas bien les Psaumes, il parle clairement des gens qui se contentent de les chanter, sans s'y arrêter vraiment, qui se trouvent dans une lecture qu'on dirait naïve et sur laquelle il va agir. Il y a donc bien une forme de savoir prérequis que le commentateur exprime par des formules du genre constat (on le sait bien), probatur (c'est un fait reconnu).

11 Plus encore que du savoir, le lecteur est supposé avoir une attitude de curiosité et d'attention, il doit avoir envie de comprendre : il est décrit comme aduertens (attentif: Claud. Don., 1, p. 12 GEORG.), diligens (plein de soin : Cassiod. Ps. 12, 1. 139; 15, 1. 32 ; 31, 1. 1 ; 106, 1.74 et 77, 1. 135 ADRIAEN) au contraire de ceux qui sont non attente legentes (ceux qui lisent sans faire attention: Claud. Don. 3, p. 294 GEORG.), parce qu'ils lisent leuiter (par amusement: Claud. Don. 1, p. 137 GEORG.). La lecture doit être studiosissima (la plus appliquée possible : Cassiod. Ps. Praef. $17 ; 46,1.1 ; 87,1.3$ ADRIAEN) et le lecteur memor doit engranger au fur et à mesure les éléments de l'explication (Cassiod. Ps.68, 1. 10 ADRIAEN) ; pour cela, il doit faire confiance au commentateur qui va le rendre intrepidus (sans peur) face aux difficultés (Cassiod. Praef. 13 ADRIAEN). On voit donc ici s'esquisser, outre la figure du lecteur idéal de Virgile ou des Psaumes, la figure de celui qui tirera pleinement parti du commentaire et qui - détail absolument essentiel - ressemble au commentateur lui-même.

\section{3 la mise en scène des interactions entre commentateur et lecteur}

12 Ainsi, l'interaction supposée entre auteur, lecteur et commentateur se trouve orientée d'une manière toute particulière, dans la mesure où l'idéal de la lecture du commentaire consiste précisément à pouvoir prendre la place du commentateur, du moins théoriquement. Nous nous trouvons ici au cœur de l'ambiguïté de la notion de lectorat dans notre corpus: quand le lecteur du commentaire se confond avec le lecteur de l'œuvre commentée, il se rapproche de la figure du commentateur, mais quand il est posé ouvertement comme lecteur du commentaire, il se réfère à l'autorité magistérielle du 
commentateur. Or évidemment, les situations ne sont pas si tranchées et la mise en scène du lectorat et de ses interactions avec le texte commenté et avec le texte du commentaire aboutit le plus souvent à un certain flou dans les rôles.

\subsection{1 le lecteur invité à commenter : quand l'élève rejoint le maître}

De manière assez régulière, le lecteur est convoqué pour émettre sa propre opinion sur le texte. On a là la mise en scène d'une discussion savante, dont on sait que les lettrés étaient friands, mais qui, dans un certain sens, reste ouverte, puisque le commentateur ne prend parti ni pour l'une ni pour l'autre des solutions. C'est au lecteur de choisir ce qui lui plaît le mieux entre deux interprétations, selon son bon plaisir (Cassiod. Ps.6, 1. 14 ADRIAEN : quod lectori placuerit eligere suffragatur), en usant de son libre arbitre (arbitrio: Cassiod. Ps. 147, 1. 167 ADRIAEN) ou en appliquant sa uoluntas (Cassiod. Ps. 147, 1. 167 ADRIAEN). Il ne semble d'ailleurs pas que la différence des termes employés marque une vraie différence dans l'opération intellectuelle demandée: il s'agit de choisir (eligere) selon des critères personnels au lecteur.

Tibérius précise cependant que l'opération doit reposer sur le iudicium du lecteur (Claud. Don. 8, p. 124 GEORG. : iudicio lectoris eligenda). Cette notion de iudicium est importante car elle inclut non seulement la notion de discernement, mais encore et surtout celle de jugement critique fondé sur le goût. Parler ici de iudicium suppose chez le commentateur la conviction que le lecteur est muni des codes du goût, autrement dit des codes culturels, linguistiques et sociaux qui lui permettent de faire le bon choix. Dans cette posture, le lecteur se fait lui-même exégète éclairé, ce qui au fond, comme Tibérius le disait à son fils, est la finalité ultime du commentaire :

Claud. Don. Proem., p. 1 GEORG.: <Post> illos qui Mantuani vatis mihi carmina tradiderunt postque illos quorum libris voluminum quae Aeneidos inscribuntur quasi quidam solus et purior intellectus expressus est silere melius fuit quam loquendo crimen adrogantis incurrere. sed cum adverterem nihil magistros discipulis conferre quod sapiat, scriptores autem commentariorum non docendi studio, sed memoriae suae causa quaedam favorabili stilo, multa tamen involuta reliquisse, haec, fili carissime, tui causa conscripsi, non ut sola perlegas, sed ut conlatione habita intellegas quid tibi ex illorum labore quidve ex paterno sequendum sit.

Après ceux qui m'ont fait connaître les poèmes du chantre de Mantoue, et ceux dont les livres ont exprimé, selon eux, le seul sens possible et le plus pur des livres de l'Enéide, il aurait mieux valu se taire que d'encourir en parlant l'accusation de présomption. Mais, je voyais que les maîtres n'apportaient rien aux élèves qui eût vraiment une valeur intellectuelle, et que les commentateurs, mus non par le zèle à enseigner, mais par le souci de leur renommée dans l'histoire, avaient certes laissé quelques passages expliqués dans un style propre à leur attirer la faveur du public, mais beaucoup d'autres dans l'obscurité. Aussi, j'ai écrit, mon fils, les notes que voici pour toi, non pas pour que tu ne lises qu'elles, mais pour que, par comparaison avec d'autres, tu comprennes ce à quoi tu dois te fier, soit dans le travail de ces gens soit dans celui de ton père.

15 Ainsi le iudicium, s'impose comme règle quand, en particulier, c'est l'auteur commenté lui-même qui laisse son lecteur dans l'expectative

Claud. Don. 12, p. 579 GEORG. : qui esse possent mixti pectorum motus aestimationi legentum poeta commisit, ut plus sentiamus quam potuit dictione concludi.

Ce que peuvent être ces 'sentiments mêlés', le poète l'a laissé à l'appréciation des lecteurs, pour que nous comprenions davantage qu'il n'a pu en contenir dans son expression. 

n'y a pas d'enjeu. Que nous pensions ce que nous voulons n'a finalement aucune importance et donc le commentateur a beau jeu de nous le laisser imaginer.

Mais dès qu'il y a de l'enjeu, il reprend la main et propose, certes, au lecteur d'exercer son iudicium, mais uniquement à titre d'exercice car finalement il a la solution, comme dans ce passage où il s'agit de savoir s'il faut rapporter l'adjectif infelix au vocatif Euryale ou au sujet de sequar, Nisus :

Claud. Don. 9, p. 241 GEORG. : iudicium hic legentis est necessarium, ut sequatur utrumque aut deligat alterum quod putauerit melius. Mihi tamen uidetur secundum animum Nisi tantum doluisse perditum, ut se potius infelicem diceret quia tardius respexit.

Ici il faut en appeler au goût du lecteur : soit il adopte les deux lectures, soit il en choisit une qu'il considère comme la meilleure. Cependant, à mon avis, la seule chose conforme à l'état d'esprit de Nisus est qu'il souffre à cause de celui dont il a perdu la trace et ainsi se dit plutôt lui-même infelix ('malheureux') parce qu'il a trop tardé à regarder en arrière.

Dans ce cas, il est clair que le choix n'est pas totalement libre : le iudicium du lecteur est certes évoqué, mais que pèse-t-il face à l'auctoritas du commentateur? Ainsi, on voit apparaître une forme de stratégie qui stimule certes la réflexion du lecteur, mais le renvoie in fine à la nécessité du commentateur, et donc à son statut inférieur face au maître.

\subsection{2 la mise en scène de la supériorité du commentateur sur le lecteur : où le maître reste le maître}

En réalité, l'existence même du commentaire sert souvent à prouver son utilité, car le lecteur seul serait perdu, s'il n'avait la présence rassurante du commentateur. Car malgré toute sa bonne volonté et son iudicium, le lecteur est assez régulièrement mis en scène comme celui qui «ne saurait pas faire tout seul». Il peut facilement être conduit à l'erreur (ad errorem uenire: Claud. Don. 10, p. 290 GEORG.) dès qu'il se trouve dans l'incertitude (incertus lector: Claud. Don. 3, p. 320 GEORG.) ou confronté à l'ambiguitas (Cassiod. Praef. 17 ADRIAEN) et à l'obscuritas (Cassiod. Ps. 24, 1. 22 ADRIAEN). Le commentateur doit donc en faire un lecteur bien armé (instructus lector : Claud. Don. 7, p. $10 ; 1,1.10$ GEORG.), averti des difficultés (admonitus: Cassiod. Ps. 3, 1. 177 ADRIAEN) en lui apportant son secours (adiuuare: Claud. Don. 11, p. 513 GEORG.). Ici l'interaction est pensée dans un rapport clairement magistériel qui légitime l'activité du commentateur.

On peut alors conclure cette partie avec cette réflexion de Tibérius qui résume, dans toute son ambiguïté, ce rapport à la fois autoritaire et libre qui s'instaure entre le commentateur et le lecteur tel que nous l'avons esquissé

Claud. Don. 3, p. 272 GEORG. : dedimus intellegendi uiam, cetera lector inueniet, quae tanta sunt uno uersu conclusa, ut haec libris explicari non possent.

Nous avons montré un chemin pour comprendre; tout le reste, le lecteur le découvrira ; le contenu de ce seul vers est tel que des livres entiers ne suffiraient à l'expliquer.

21 On voit donc que se construit ici une forme d'interaction très particulière, où le lecteur est à la fois en position d'élève et de chercheur, comme une sorte de maître virtuel que le commentaire aiderait à progresser dans la nécessaire compréhension du texte, préambule à son explanatio. Mais en même temps, ce lecteur n'est pas une page blanche sur laquelle le commentateur écrirait son cours; il est attendu de lui qu'il possède déjà les références 
culturelles, le code qui permet de décrypter le commentaire et par-delà le commentaire le texte commenté. On est donc très loin d'un commentaire d'initiation, et le commentaire scolaire apparaît comme une forme de "discours d'initiés ", entre spécialistes plus ou moins avancés mais pourvus du même substrat culturel ${ }^{5}$.

\section{Donat et son lecteur}

Comme on le voit dans ce qui précède, l'opération désignée par le verbe legere recouvre plusieurs activités connexes mais différentes, comme nous allons le voir cette fois spécifiquement chez Aelius Donat commentant Térence ${ }^{6}$.

\section{1 les diverses acceptions de legere}

\subsection{1. lire $=$ déchiffrer}

Legere c'est primitivement lire des lettres pour les assembler en mots. Evidemment, au stade de compétence où en sont les lecteurs de Donat, il ne va pas s'agir de remarques élémentaires mais de notations plus subtiles destinées par exemple à opérer des differentiae entre graphies proches ou identiques : ainsi chez les Anciens, qui peut valoir cui :

Don. And. 167, 1 : et cui per q ueteres scripserunt

les anciens écrivaient aussi le datif cui avec un q.

Ou encore :

Don. And. 81, 3 : VT VNA ESSET M. et producte legitur esset, ut cibum capiat, et correpte, ut alibi...

esset se lit aussi bien avec un e long, au sens de 'prendre de la nourriture', qu'avec un e bref <au sens du verbe 'être'> comme ailleurs....

Dans ce sens, lire c'est alors, techniquement, reconnaître des graphèmes, les distinguer les uns des autres, repérer des signes auxiliaires (notae, abréviations, marques de quantité, etc.), activité en relation avec la discipline de l'orthographia qui fleurit notamment à l'ère tardo-antique. Ainsi peut-on supposer que, pour le deuxième exemple cité, le lecteur avait, pour distinguer à l'œil èsset de ěsset, une aide graphique, le macron ou l'apex (ou leur absence), dans la mesure où les deux verbes sont ici plausibles (« pour qu'il mange avec eux » vs " pour qu'il soit avec eux »). Et la connaissance des signes de ponctuation ainsi que, en cas d'absence, la manière de les suppléer, font partie de cette discipline qu'est la lecture avancée :

Don. Ad. 693, 2: QVID CREDEBAS DORMIENTI HAEC TIBI CONFECTVROS DEOS incerta distinctio, nam et per se quid et quid credebas legi potest.

QVID CREDEBAS DORMIENTI HAEC TIBI CONFECTVROS DEOS ponctuation incertaine car on peut lire aussi bien quid? tout seul que quid credebas ?.?

\subsection{2. lire $=\mathrm{s}^{\prime}$ informer}

Lire c'est aussi apprendre quelque chose que l'on tire d'un livre :

Don. Ph. 49 3: VBI INITIABVNT legitur apud Varronem initiari pueros Eduliae et Poticae et Cubae, dis edendi et potandi et cubandi...

VBI INITIABVNT On lit chez Varron que les enfants étaient initiés à Edulia, Potica et Cuba, déesses de la nourriture, de la boisson et du coucher... 

(« apprendre par la lecture »).

\subsection{3. lire $=$ réciter}

Lire, dans ce monde antique où la lecture silencieuse n'a pas ou presque pas cours, c'est aussi bien sûr proférer à haute voix un texte écrit de manière à en faire naître toutes les nuances, le réciter ${ }^{8}$. Ainsi quand il est question de tonalité, comme dans ces passages :

Don. Ph. 169, 1: QVOD HABES ITA VT VOLVISTI uoluisti subdistinguendum est et sic legendum uxorem $\dot{\varepsilon} \mu \varphi \alpha \tau \imath \kappa \omega ́ \tau \varepsilon \rho o v$.

QVOD HABES ITA VT VOLVISTI Il faut ponctuer après uoluisti et lire ainsi uxorem avec une certaine emphase.

Lire relève alors de la pronuntiatio, qui est plus affaire de rhéteur que de grammairien (voir Cic. Inv. 1, 9 ; Quint. Inst. 1, 11, 14).

\subsection{4. lire = éditer}

Lire, c'est aussi adopter telle leçon plutôt que telle autre dans un manuscrit qu'on a sous les yeux. L'activité relève alors clairement de l'ecdotique et de la critique textuelle. On voit ainsi le grammairien signaler deux variantes morphologiques plausibles (Don. Eun. 1022 , 2) ${ }^{9}$. Il peut aussi proposer deux lectures lexicalement différentes mais contextuellement acceptables toutes les deux :

Don. And. 344, 2 : habeo 'inueni'. legitur et abeo, ut merito illi dicatur 'resiste'.

habeo équivaut à 'j'ai trouvé'. On a aussi la leçon abeo ('je m'en vais'), ce qui rend plausible qu'on lui dise resiste ('attends') ${ }^{10}$.

Il peut aussi athétiser telle leçon, fautive ou incohérente ou donner une préférence à telle variante (Don. And. 653, 2, et Don. Hec. 670, 2 par exemple ${ }^{11}$ ).

Les exemples de ce sens de legere en lien avec l'activité d'édition critique sont nombreux dans le commentaire à Térence avec notamment le schème récurrent legitur et $x$ (« on lit aussi $x$, on connaît aussi la leçon $x »$ ), car Donat collationne effectivement plusieurs manuscrits et compare et critique aussi les leçons de plusieurs commentaires antérieurs (ceux de Probus, Asper, Evanthius, peut-être Nigidius). Dans ce cas, même l'actif legere s'oriente vers le sens de "prendre telle leçon textuelle » et glisse vers le sens paradoxal d' « écrire » ou celui d' « éditer » :

Don. Ad. 574, 2 : VBI EO VENERIS CLIVVS DEORSVM VERSVM EST HAC TE PRAECIPITATO hoc lentius quidam. aliqui clauus legunt, aliqui cliuus, ut sit praecipitato' cito descende'.

VBI EO VENERIS CLIVVS DEORSVM VERSVM EST HAC TE PRAECIPITATO cette réplique est en aparté selon certains. Certains lisent (=éditent) clauus (clou), certains cliuus en sorte que te praecipitato signifie 'cito descende' (dépêche-toi d'y descendre) ${ }^{12}$.

Voir aussi l'expression sunt qui legant (il y en a qui éditent telle leçon) dans And. 618, 2, Hec. 590, 3, Ph. 485, 2.

\subsection{Lire et processus de lecture}

Les différents emplois de legere influent sur ceux de son dérivé lectio, que Donat connaît aussi. Lectio c'est ainsi, en référence aux sens 1 et 3 du verbe de base, l'action de déchiffrer et d'oraliser la lecture : 
Don. Ad. 324, 2: ANIMAM RECIPE quod in lectione gestu ostendi minime potuit, id ex uerbis Sostratae ostenditur in Geta.

ANIMAM RECIPE ce qui à la lecture ne pouvait guère être montré par une gestuelle est montré, par les mots de Sostrata, à propos de Géta ${ }^{13}$. voit ainsi le verbe legere (dans sons sens «éditer telle variante textuelle» ou «prononcer ») voisiner avec son préverbé intellegere ${ }^{14}$ dans plusieurs passages. Par exemple :

Don. Hec. 424, 3 : (...) nam qui iungunt et sic legunt, errant et non intellegunt. ceux qui ne ponctuent pas et éditent ainsi le texte se trompent et ne comprennent pas.

$$
\begin{aligned}
& \text { Mais cette relation de lire à comprendre se fait dans les deux directions, dans un aller- } \\
& \text { retour permanent : c'est parce que nous déchiffrons un texte que nous le comprenons et } \\
& \text { que nous pouvons l'interpréter (au sens théâtral); mais dans l'autre sens c'est parce que } \\
& \text { nous comprenons une situation dramaturgique que nous pouvons choisir telle variante } \\
& \text { textuelle à l'exclusion de telle autre }{ }^{15} \text {. Lire c'est interpréter finement le sens de ce qui est } \\
& \text { écrit et les intentions de l'auteur, quitte à amender un texte corrompu pour retrouver } \\
& \text { l'original. Lire, c'est récrire éventuellement : intellegitur... ergo legendum est (Don. Eun. 315), } \\
& \text { "on comprend, donc il faut lire ainsi ». }
\end{aligned}
$$

\section{3 le lecteur}

Partant, le lecteur idéal pour Donat est celui qui sait legere : il sait donc, dans un ordre croissant de compétence, déchiffrer (inutile d'insister sur cette évidence), il sait chercher une information dans un ouvrage, il doit savoir aussi prononcer un texte qu'il lit en lui donnant tous ses effets de sens, il doit se montrer sélectif dans le choix des variantes et il doit être intelligent. 
Ce lecteur, Donat ne l'évoque guère directement. Alors que le verbe legere est très présent dans son commentaire, le mot lector n'apparaît que cinq fois. Dans un cas, il s'agit d'opposer le lecteur à l'acteur, celui qui lit seulement à celui qui donne au texte sa dimension théâtrale ${ }^{16}$. Dans trois autres cas, Donat nous fait part d'une intention de l'auteur par rapport à son lecteur. Dans la préface aux Adelphes, il dit quelque chose du choix du titre Adelphoe. Ailleurs on voit Térence créer une forme de suspens; enfin, Térence s'explique, voire s'excuse auprès de son lecteur sur une anomalie caractérologique.

Mais il y a là un fait capital : dans ces trois cas, l'interaction se fait directement du poète à son lecteur : le lecteur évoqué est le lecteur de Térence, non celui de Donat. La dernière occurrence nous met sur la piste du lecteur de Donat. Nous prenons un contexte un peu long, car il semble qu'il soit important :

Don. And. Praef. II, 3 : Difficile est diuisionem actuum in Latinis fabulis internoscere obscure editam: causam iam dudum demonstrauimus unde autem aut quomodo quamuis aegre tamen intellegi distinguique possint, est operae pretium discere. principio dicendum est nullam personam egressam quinquies ultra exire posse; sed illa re plerumque decipimur, quod personam, cum tacuerit, egressam falso putamus, quae nihilo minus in proscaenio tacens loquendi tempus exspectat. est igitur attente animaduertendum, ubi et quando scaena uacua sit ab omnibus personis, ita ut in ea chorus uel tibicen obaudiri possint. quod cum uiderimus, ibi actum esse finitum debemus agnoscere. confundit saepe lectorem illud , quod persona in superiore scaena desinens et in proxima incipiens loqui non intellegitur ingressa, quod ipsum experientes statim diiudicant de rerum ac temporum quantitate. potest enim fieri, ut et ingressa sit et egressa, quam praue credimus de proscaenio non recessisse.

Il est difficile de connaître clairement la division des actes dans les pièces latines, car elle est masquée, pour une raison que nous avons déjà montrée. Mais sur quels critères et comment il est possible (bien que ce soit difficile) de la comprendre et de la repérer, cela vaut la peine de l'apprendre. Commençons par dire qu'un personnage déjà entré cinq fois sur scène ne peut plus y revenir. Souvent nous nous trompons en pensant à tort qu'un personnage, du fait qu'il était silencieux, vient d'entrer en scène, alors qu'il attend sans parler à l'avant-scène le moment pour lui de donner une réplique. Il faut donc observer attentivement où et quand la scène se vide de tous les personnages, en sorte que l'on peut y écouter le chœur ou le flûtiste. Quand nous voyons cela, nous devons reconnaitre qu'il y a là la fin d'un acte. Souvent le lecteur est abusé par le fait qu'un personnage donne la dernière réplique de la scène précédente et la première de la scène suivante sans se rendre compte qu'il est sorti et rentré dans l'intervalle, ce que les experts déterminent aussitôt grâce à l'enchaînement des événements et à la durée écoulée. Car il peut arriver qu'un personnage soit sorti puis entré alors qu'on croyait bêtement qu'il n'avait pas bougé de l'avant-scène ${ }^{17}$.

Contentons-nous de constater avec Donat que le lecteur (lectorem) se laisse abuser ( confundit) facilement par de fausses impressions ; seul un expert (experientes) peut l'aider à y voir plus clair. Il s'agit d'un lecteur de Térence, là encore, puisque l'erreur qu'il commet porte bien sur la structure de la pièce qu'il a sous les yeux. Mais l'expert susceptible de le mettre sur la bonne voie, c'est le commentateur de Térence, c'est Donat lui-même. Le lecteur brut est incapable de bien lire son Térence; en revanche, le lecteur de Donat acquiert cette compétence. Le lecteur de Donat est un lecteur de Térence in progress. Il est remarquable que le lecteur abusé dont nous parle le grammairien soit évoqué dans la Préface du commentaire à L'Andrienne, la première pièce commentée par Donat, donc au tout début de ce grand ouvrage. Il est ensuite assez aguerri pour comprendre les effets que Térence a créés directement à son intention. 

Térence s'adresse directement doit avoir des compétences de niveau universitaire. Par exemple (dans la préface aux Adelphes citée ci-dessus), le poète dit que la pièce s'intitule Adelphoe et c'est sa seule explicitation. Le message que prête Donat à Térence est entièrement implicite: il souhaite faire savoir quelque chose au lecteur (cupit scire lectorem) mais sans le renseigner effectivement. C'est à lui seul, comme un grand lecteur qu'il est devenu grâce à Donat, de comprendre que le titre en grec affirme programmatiquement son appartenance au genre de la palliata, de savoir aussi que Adelphoe signifie Fratres (ce que Térence ne dit pas explicitement : c'est Donat qui le dit dans sa Préface), ce que peut-être un lecteur romain de base ne sait pas forcément ${ }^{18}$, de déduire que si la pièce s'intitulait Fratres, elle serait invariablement une togata et non une palliata. Bref, le lecteur à qui Térence fait savoir tout cela sans le dire, c'est le lecteur qui est passé par le commentaire de Donat.

\section{Conclusion}

49 La pragmatique du commentaire donatien, comme sans doute de tout commentaire antique suivi, se constitue donc sur une situation d'énonciation complexe. Préexistent un auteur, Térence, et un texte, une comédie de Térence ; est donc attendu en complément un lecteur, selon la chaîne simple ego scribo; tu lege de Diomède (GL 1, 389, 3). Mais cette relation est améliorée, voire ne prend sa réelle dimension, que si le lecteur pressenti lit en préalable (ou au mieux en même temps) un autre texte, celui du commentateur. La chaîne (chrono)logique modifiée est alors plutôt: ego Terentius scribo; ille Donatus de me scribit ; tu lector Donatum lege et, postea, me legere poteris : Térence a écrit pour qu'un jour (lointain) Donat en fasse l'exégèse. Le lecteur (au sens plein) de Térence vit, au plus tôt, au quatrième siècle après Jésus-Christ, en sorte que la chaîne en question n'est plus ni logique ni chronologique.

Cette place prépondérante du commentateur comme intermédiaire entre le poète et son lecteur explique sans aucun doute le destin de la transmission du texte même du commentaire donatien: d'abord pensé comme un livre avec son auctorialité propre (Donat dit ego pour son propre compte, voir par exemple Don. Ad. 259, 2 : sed ego puto fratrem non subdistinguendum), il finit par rendre le texte térentien brut impropre à la lecture. On se met à démembrer le traité de Donat pour que les gloses du grammairien accompagnent désormais directement le texte de la comédie, centré sur la page et entouré dans toutes ses marges des scholies donatiennes qui rendent le lecteur plus intelligent. A ce stade (et avant qu'on démembre à nouveau ces gloses pour tenter de les reconstituer en traité autonome), le texte des deux auteurs étant fusionné, le lecteur de Térence et le lecteur de Donat sont désormais une seule et même personne. 


\section{BIBLIOGRAPHIE}

ASTEll Ann W., " Cassiodorus's 'Commentary on the Psalms' as an 'Ars rhetorica' ", Rhetorica 17, no 1 (1999), 37-75.

BUREAU Bruno, «Quelques réflexions sur la notion de littérarité à partir de l'édition numérique de commentateurs anciens », Interférences [En ligne], 6 | 2012, mis en ligne le 08 mars 2013, URL : http://interferences.revues.org/186.

GOULET-CAZÉ Marie-Odile (dir., avec la collaboration éditoriale de Tiziano DORANDI, Richard GOULET, Henri HUGONNARD-ROCHE, Alain LE BOULLUEC, Ezio oRNATO), Le commentaire entre tradition et innovation, Actes du colloque international de l'Institut des traditions textuelles (Paris et Villejuif, 22-25 septembre 1999), Paris, Vrin, 2000, « Bibliothèque d'Histoire de la Philosophie ».

KASTER Robert, Guardians of Language : the Grammarian and Society in Late Antiquity, Berkeley, University of California Press, 1988.

HOLTZ Louis, « Les manuscrits latins à gloses et à commentaires de l'Antiquité à l'époque carolingienne », in QUESTA Cesare et RAFFAELLI Renato (dir.), Atti del Convegno internazionale « Il libro e il testo »(Urbino, 20-23 settembre 1982), Urbino, Pubblicazioni dell'Università di Urbino, Science umane 1, 1984, 141-166.

HOLTZ Louis, « Le rôle des commentaires d'auteurs classiques dans l'émergence d'une mise en page associant texte et commentaire (Moyen-Âge occidendal) », in GOULET-CAZÉ Marie-Odile (dir., avec la collaboration éditoriale de Tiziano DORANDI, Richard GOULET, Henri HUGONNARD-ROCHE, Alain LE BOULLUEC, EzIo ORNATO), Le commentaire entre tradition et innovation, Actes du colloque international de l'Institut des traditions textuelles (Paris et Villejuif, 22-25 septembre 1999), Paris, Vrin, 2000, « Bibliothèque d'Histoire de la Philosophie », 101-118.

NICOLAS Christian, « À la recherche des fins d'acte et des fins de scène dans les comédies de Térence lues par Donat ", in Bureau Bruno et Nicolas Christian (dir.), Commencer et Finir. Débuts et fins dans les littératures grecque, latine et néolatine, Lyon, Editions Lyon 3, 2008, 595-620.

PIROVANO Luigi, Le Interpretationes Vergilianae di Tiberio Claudio Donato : problemi di retorica, Roma, Herder, 2006.

SCHLIEBEN Reinhard, Christliche Theologie und Philologie in der Spätantike. Die schulwissenschaftlichen Methoden der Psalmenexegese Cassiodors, Berlin/New York, De Gruyter, 1974.

SQUILLANTE SACCONE Marisa, Le Interpretationes Vergilianae di Tiberio Claudio Donato, Napoli, Società Ed. Napoletana, 1985.

VALETTE-CAGNAC Emmanuelle, La lecture à Rome : rites et pratiques, Paris, Belin, 1997.

\section{NOTES}

1. Sur le commentaire antique comme genre, voir Goulet-Cazé et alii, 2000. Sur la question spécifique de la lecture du commentaire et de la construction auctoriale de la figure du commentateur, voir Bureau 2012. Sur la lecture à Rome et ses implications, voir Valette-Cagnac, 1997. 
2. Sur ces sujets, voir HOLTZ, 1984 et HoLTZ, 2000.

3. Nous lisons Tiberius Claudius Donat dans l'édition suivante : Tiberi Claudi Donati ad Tiberium Claudium Maximum Donatianum filium suum. Interpretationes Vergilianae ed. Henricus Georgii ; Adiectae sunt Vitae Vergilianae quas recensuit Iacobus Brummer. Ed. stereotypa editionum anni 1905 (1969). Sur Tiberius et son commentaire, voir SQUILLANTE 1985 et PIROVANO 2006.

4. Sur ce texte, voir par exemple ASTELL 1999 et SCHLIEBEN 1974. Nous lisons Cassiodore dans l'édition ADRIAEN (CCL), Magnus Aurelius Cassiodorus, Expositio psalmorum I-LXX, et LXXI-CL, Turnhout, 1958.

5. A ce sujet, on se reportera à l'indispensable synthèse de KASTER, 1988.

6. Nous lisons Donat dans l'édition de Paul Wessner (Teubner 1902); la traduction est, sauf remaniement ad hoc, celle qu'on trouve sur notre site Hyperdonat. Voir sur hyperdonat.humanum.fr.

7. On peut donc comprendre : «Quoi ? Tu croyais qu'en dormant etc. ?» ou «Qu'est-ce que tu croyais? Qu'en dormant etc. ?».

8. La prégnance de la lecture oralisée se voit dans cette chaîne (chrono)logique de Diomède : GL 1, 389, 3 ego scribo, tu lege ; ego lego, tu audi (moi j'écris, toi, lis ; moi je lis, toi, écoute). Si lire est le complémentaire d'écrire, on voit qu'il a aussi lui-même pour complémentaire entendre. On écrit donc pour qu'à l'arrivée quelqu'un entende le message ; lire est le centre d'un processus à trois actants : un scripteur, un lecteur (qui est un récitant), un auditeur.

9. Il s'agit en l'espèce d'un accord au pluriel pour le sens uterque edent, ce qui relève de la syllepse de nombre. Les deux leçons uterque edent et uterque edet sont plausibles.

10. De fait les deux leçons se lisent dans la tradition térentienne, ce qui se comprend aisément si le scribe écrit sous la dictée : habeo et abeo, parfaits homonymes à l'oreille, sont ici tous les deux plausibles (" je m'en vais » ou « j'ai <la solution> », "ça y est »). Dans les deux cas, Dave s'apprête à se retirer et la réplique de Pamphile «Dave, attends, arrête » peut s'enchaîner aussi bien sur l'une que sur l'autre solution.

11. On a dans ce dernier cas, Hec. 670, 2, une remarque d'ecdotique (si ipsa legeris vs si ipse...) qui a des ramifications dramaturgiques. Si la leçon est ipsa pater, pater est forcément un vocatif et, donc, la réplique se fait à haute et intelligible voix, Pamphile s'adressant en réponse directe à son père : «Un bébé qu'elle-même, père, a négligé, moi je l'élèverais ?»; si en revanche la bonne leçon est ipse, alors le groupe ipse pater désigne le père biologique et, comme sa non-paternité est un secret que Pamphile ne souhaite pas révéler, la réplique doit être en aparté : «Un bébé que son propre père a négligé, moi je l'élèverais?». La réplique de Lachès «quid dixti? » peut s'enchaîner aussi bien sur la réplique à voix haute que sur la réplique en aparté, si c'est un aparté à moitié audible (que Donat appelle une murmuratio, un grognement), comme cela est fréquent au théâtre. Donat opte pour la solution de la réplique à voix haute, avec ipsa et pater au vocatif dans la scholie 3, puis pour l'aparté dans la scholie 4. C'est la preuve que nous avons là des scholies d'auteurs et d'époque différents, celle de Donat et celle de commentateurs de Donat et qu'il est bien souvent impossible de savoir ce qui revient au commentaire originel.

12. Pour un éclaircissement (relatif) sur cette scholie, voir notre édition et nos notes sur Hyperdonat ad loc. en suivant le lien http://hyperdonat.huma-num.fr/editions/html/ DonAde.html\#574.

13. Comprendre: Géta arrive essoufflé pour une scène comique de servus currens. Il ne peut pas aligner deux mots. Sur scène, l'acteur montrait toute la panoplie gestuelle et mimique du servus currens. Mais à la lecture, faute de voir l'essoufflement, nous devons le déduire (puis le reproduire dans la lecture oralisée) à partir de la réplique de Sostrata.

14. Les deux verbes, qui se sont chacun spécialisés au point que leur rapport morphologique n'est plus très sensible, sauf retour métalinguistique volontaire, partagent étymologiquement le sème 'choisir, sélectionner', d'où, selon les langues qui utilisent la racine *leg-, des acceptions comme 'cueillir', 'lire', 'dire', 'choisir'. 
15. Par exemple ce que nous déduisons du texte nous invite à voir là un aparté plutôt qu'une réplique à pleine voix, ce qui impose ipse plutôt que ipsa dans l'exemple de Hec. 670, 2 ci-dessus.

16. Ph. 211, 1 SATIN SIC EST hic locus actoris magis quam lectoris est: ostenditur enim conari ad simulandam audaciam, quo magis ridicule formidulosa persona est, «ce passage relève davantage du théâtre joué que du théâtre lu : Antiphon est présenté en train d'essayer de feindre l'audace, ce qui rend le personnage encore plus ridiculement peureux ».

17. Sur la question des cinq actes, voir NICOLAs 2008.

18. On dirait sûrement la même chose de titres de palliatae impliquant un degré de bilinguisme important, comme Synapothnescontes (cité par Térence dans le prologue des Adelphes, 5, aussitôt explicité en Commorientes) ou le térentien Heautontimorumenus.

\section{RÉSUMÉS}

En partant d'une comparaison avec les commentaires de Claudius Donat à Virgile et de Cassiodore au Psautier, on tente de tracer le portrait du lecteur d'un commentaire antique suivi comme celui de Donat à Térence et l'on se demande quelles opérations complexes recouvre l'acte signifié par le verbe latin legere.

After a comparison with Claudius Donatus' Interpretationes Vergilianae and Cassiodorus' Comment on Psalms, the paper deals with the figure of the reader of a Late Antiquity comment such as Aelius Donatus' work on Terentius and wonders what kind of complex actions are involved by the Latin verb legere.

\section{INDEX}

Mots-clés : commentaire tardo-antique, Donat, lecture, apprentissage, Térence

Keywords : Late Antiquity Commentary, Donatus, Reading, Learning, Terentius

\section{AUTEURS}

\section{BRUNO BUREAU}

Professeur de langue et littérature latines à l'Université Jean Moulin Lyon 3 et membre de l'UMR HiSoMA. Avec C. Nicolas, il a publié dans la collection Commentario des Belles Lettres L'Eunuque de Térence (2015) et est le concepteur et éditeur scientifique du site Hyperdonat.

\section{CHRISTIAN NICOLAS}

Professeur de langue et littérature latines à l'Université Jean Moulin Lyon 3 et membre de l'UMR HiSoMA. Avec B. Bureau, il a publié dans la collection Commentario des Belles Lettres L'Eunuque de Térence (2015) et est le concepteur et éditeur scientifique du site Hyperdonat. 